\title{
PARTICLE ACCELERATION MY MAGNETOSONIC WAVES IN A CORONAL LOOP
}

\author{
J.-F. de La Beaujardière and E. G. Zweibel \\ Department of Astrophysical, Planetary and Atmospleric Sciences \\ University of Colorado, Boulder, CO 80309-0391
}

\begin{abstract}
A model for the acceletation of electrons in a flaring coronal loop is described. The mechanism is stochastic acceleration by resonant interactions with a spectrum of compressive magnetosonic waves. Current results of test particle calculations examining the feasibility of this model ane presented.
\end{abstract}

\section{Introduction}

The impulsive plat. of solar llares is characterized by strong emission of hard $x$-rays and of microwaves. The duration of this phase is brief $(\approx 1 \mathrm{~min})$ and has a rapid rise time $(\approx 5 \mathrm{sec})$. We are investigiting a model for the acceleration of the electrons responsible for the hat $x$-ray and microwave emissions. The mechanism we are considering is stochastic acceleration by magnetosonic turbulence exciten hy the llare primary energy release (Kulsrud and Ferrari 1971; Meh1, _e 1980; Achterberg 1981; Forman, Raunaty and Zweibel 1986).

Our calculations differ from previous work in two respects: First, we assume that the turlualence is composed of the wave modes of a cylindrical loop. Second, rather than calculating the acceleration rate from quasilinear theory, we integrate particle orbits directly using the guiding center approximation.

\section{Magnetosonic Waves in a Coronal Loop}

We model a coronal loop by as staight magnetized cylinder of length $L$ and radius a. The cylinder is embedded in an infinite medium of differing density, temperature and magnetic field strenthts. We require pressure batance as an equilibrium condition. 'The effects of gravitational stratification are neglected.

A dispersion relation for compressive magnetosonic waves in a magnetic fluxtube can be derived from the equations of ideal magnetolydrodynamics (MIID). The dispersion relation can be solved analytically in the limit of high frequencies, and mumerically otherwise.

The character of the waves differs greatly depending on the lieguency range under consideration. Certain modes are evanescent, having significant amplitude only neal the tube wall at $r=a$. Other modes are oscillittory throughout the tube. The highest-frequency waves can propagate outside the loop, catusing wave energy to be lost from the system. This leakage maty be related to observations of sequential, spatiallyseparated flares, because it suggests that a disturbance could propagate from one loop to others nearby.

\section{The Equation of Motion}

In the absence of watves, particle motion consists of translation parallel to the ambient field combined with gyration perpendicular to the ficldlines it the cyclotron freguency $\Omega \equiv e B /$ rne. For precesses occuring over timescales and lengthscales long compared to the gyromotion, the particle's magnetic moment $\mu \equiv m v_{1}^{2} / 2 B$ is an adiabatic invariant. The particle can be confined in the comnal loop by the magnetic mirror force $-\mu \nabla B$, and can execute repeated bounces from one end of the trop to the other.

The magnetosonic waves considered herein create an a.c. elcctric field component $E_{1 z}$ directed along the loop axis. This electric ficld can accelerate particles. The pinysical origin of $E_{1 z}$ is as follows: A perturbation $B_{1}$ in the magnetic field creates a mirror acceleration $-(\mu / m) \nabla B_{1}=-v_{\perp}^{2} \nabla B_{1} / 2 B$, wlich is typically larger for electrons (their mean $v_{\perp}$ being greater). This preferential acceleration causes a charge separation, which creates an electric field.

The equation of motion for an electron in the presence of a single wave is (Barnes 1007)

$$
\frac{d^{2} z}{d l^{2}}=\left(\frac{\bar{\mu}-\mu}{m}\right) \frac{\partial B_{1 z}}{\partial z}
$$


where $i s=\kappa T / B_{0}$ is the mean magnetic monent of the distribution ( $\kappa$ is Bultzmann's constant), and the right hind side is summed over all the wave modes in the system. We integrate this equation of notion anuserically using a test particle approach.

\section{The Ifamiltonian Formulion}

One can derive the equation of motion (1) from a Hamiltonian which, in the rest frame of the wave, is

$$
H-\frac{p^{2}}{2 m}+(1+-\mu) H_{U}\left[1+\frac{1}{2} b \sin \left(k_{z} z\right)\right]+\frac{1}{2} m v^{2}
$$

whete $b$ is popentionial to the wave amplitude and $v_{\psi}$ is the parallel phitse speed of the witve. Contours of this llanilusuinu are sketched in lig'ure 1.

The: sephtolrix divides the position-numentum phase plane $(z, p)$ into open and closed particle orbits. l'aticles within the sepalatrix contuar are called resonant particles and are trapped in the wave potential. It is these patticles which at te most important for our accelerition mechanism. The hald-height of che separatrix is given liy

$$
\Delta p_{r}=\left(2 m B_{u}\left|\mu^{*} \ell\right|\right)^{1 / 2}
$$

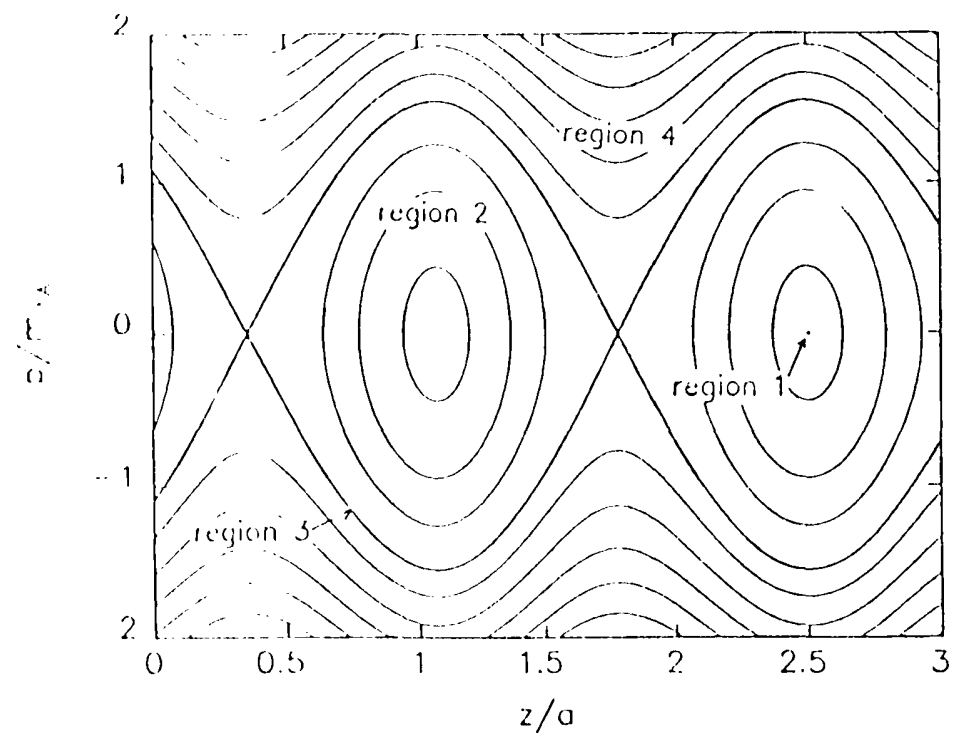

Figure 1 Contours of the Hamiltonian If in the wave rest frame. The scale on the ordinate is arbitrary. Region 1: Minima of the witve potential; particle is stationary in the wave reference franc. Region 2: Trapped region; particle oscillates about the wave speed. Region 3 (thicker contour): The separatrix; particle migrates towarls $\mu=0$ line. Region 4: Untrapped region; pallicle moves faster or slower than the wave.

\section{Particle Acceleration}

If a parlicle interacts with many waves whose separatrices overlap (in the fashion described below), the piaticle can muve from one wave potential to another, thus diffusing in velocity space. The mechanism for electron acceleration we are investigating relies on this diffusion.

The basic idea is as follows: Electruns in the loop will initially be in resonance with those waves having phise speed " higher phase velocity; the speed of the particles can thus increase up to the maximum $v_{\psi}$ in the system. One can visualize this process as a particle "climbing a ladder" of overlapping wave potentials. Note that thermal electrons are accelerated; no injection of energetic particles is required.

'l'wo waves have overlapping polcutials when they are close enough in phase speed, and of sufficiently luge anplitude, that the areas enclosed in the $(z, p)$ plane by their separatrices intersect. This overlap criterion is not strictly correct, because each separatrix is calculated in its own rest frame and because there exist higher order resunances (due to wave-wave interactions) between the primary ones. Ilowever, we have found his criterion to be a use?ul dianostic tool in predicting the onset of chaos. 


\section{Chaos}

Particle trajectories in overlapping wave polentials are chiotic. This means, for example, that the urluits of particles with nearly identical initial conditions will diverge signilicantly with time. An eximple of this phenomenon is depicted in fignre 2, which shows the trajectories of three particles with slightly different initial velocities (the velocity clifferences are about $10^{-4}$ and $10^{-3}$ of the electron theminal speed). The calculated orbits differ significintly despite their initial proximity. This effect occurs regardhess of the sinallness of the integration timestep.

Because particle trajectories are chiotic, the precise trajectory of a single test particle is not the most physically uneiningful yuantity one can calculate. More important, for example, is the final state of an ensemble of test particles.

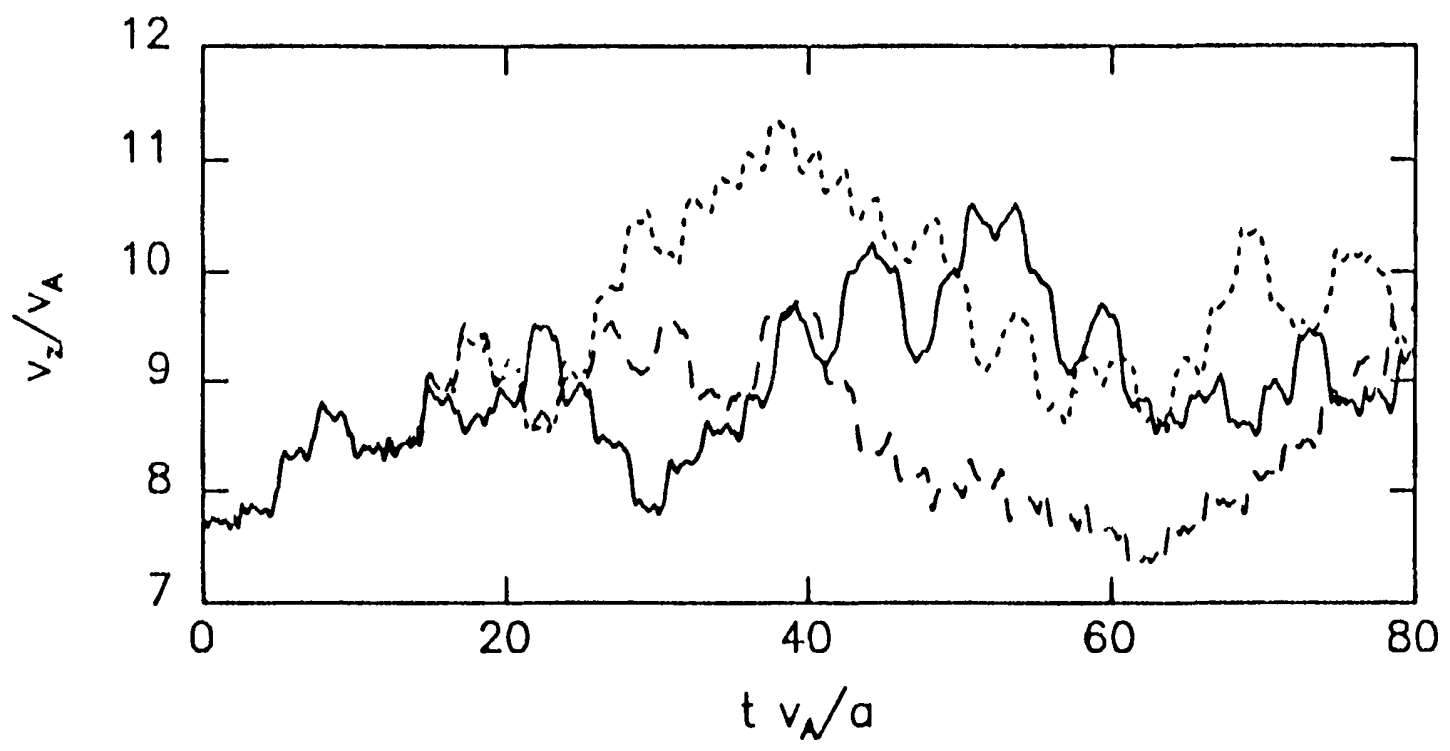

Figure 2 Thiajectories of particles with slightly different initial velocities. Solicl: $v_{z i}=7.74500 v_{A}$. Dotted: $v_{z i}=7.7 .501 v_{A}$. Dislied: $v_{z i}=7.74510 v_{A}$.

\section{Preliminary Results}

We have begun a series of supercomputer calculations to determine the statistical beliavior of electrons in the wave system. In these test-particle sinulations we employ the guiling center approximation (in which the gyromotion of the particles is neglected), and we directly integrate the equation of motion (1). The calculations are still in the preliminary stages and program development is not yet complete, but some information has been gleaned from them.

A requirement of this acceleration mechanism is that the waves in the system overlap sufficiently to cover all of the relevant velocity range (from thermal energies to $\sim 10-100 \mathrm{keV}$ ). We have found that this requirement inposes certain restrictions on the wave and particle parameters. Wave overlap depends on the separatrix half-height (ec. 2), which is a function of the wave aneplitude $b$ and of the particle's magnetic moment $\mu$. Wave amplitudes are limited, however, by our use of linearized MHD to derive the dispersion relation. Consequently, only particles with large enough $\mu$ can diffuse thronghout the entire velocity range. Pitch-angle scattering is therefore a necessary component, of this mechanism.

Some of the waves used in these simulations are damped by leakige out of the tube, as discussed in Section II. The e-folding times range from 0.4 seconds to $>2$ seconds. It is necessary, therefore, that waves be introduced into the system over some finte period, rather than instantanerusly, to maintain the accelerating spectrum.

We lave performed several simulations with the current version of the program. 'These are characterized ty small particle numbers $(\approx 100)$ and short elipsed times (1.2 seconds). Modest particle acceleration has 
becu olselved, lut not to the energies required for yolar flate impulsive phase emission. Figure 3 is a histogram of maxinum particle encegies reaclied within 1.5 seconds by a group of particles all initially al the thermal energy; the overall energy of the distribution has clearly increased.

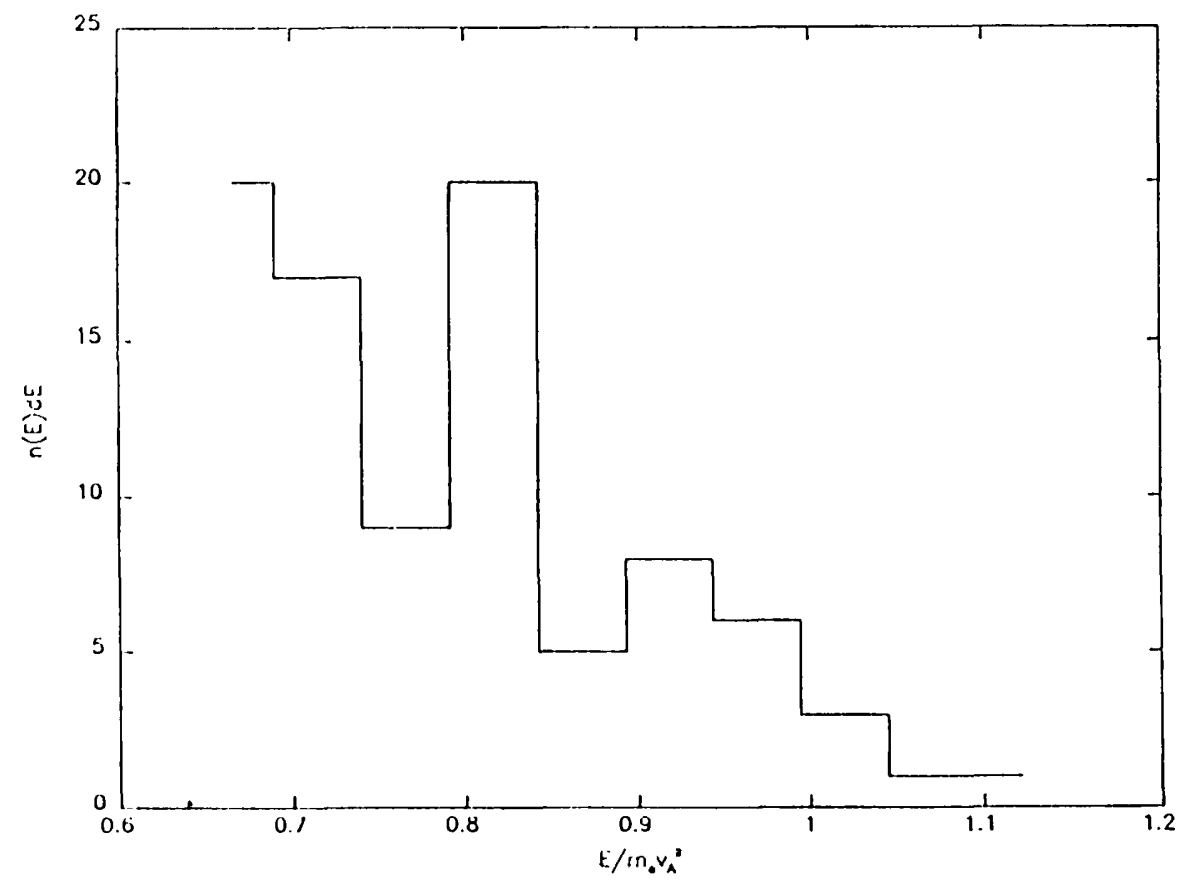

Figure 3 llistogran of maximum particle energies reached within 1.5 seconds. Initial particle energy $E_{i}=k T=0.637 m_{e} v_{\lambda}^{2}$. The simulation contained 90 patticles, with initial pitch angles between 0 and 89 degrees.

\section{Conclusion}

We have presented prediminary results of a model for electron acceleration in a flaring solar coronal lom. We suggest that a spectrum of compressive magnetosonic waves will be established in the loop by the primaty energy release event (e.g., magnetic reconnection), and that particles will be accelerated via resontant interactions with these waves.

In future work this model will be investigated more fully. Simulations with langer particle samples and longer run times, and including ellects such aty particle scattering by very high frequency waves and proluneed intruduction of wave energy, will be considered. It is clear even from the preliminary examples presented here that upwards diffusion in energy does occur. The efficiency and timescale of this acceleration mechanism remain to be determined, but his mechanism is a likely candidate for producing at least some of the hard $x$-ray and microwave emitting electrons.

Achterberg, A. 1981, Astrun. Astrophys., 97, 259.

\section{References}

Barnes, A. 1907, Pliys. Fluids, 10, 2427.

Dulk, G. A. and Dennis, J3. R. 1982, Ap. J., 260,875.

Forman, M. A., Ramaty, R., and Zweibel, E. G. 1986, in Physics of the Sun, ed. P. A. Sturrock (Dordrecht: Reidel), p. 249.

Kulsrud, R. M. and Ferrari, A. 1971, Astrophys. Space Sci., 12, 302.

Melrose, D. B. 1980, Plasma Astrophysics (New York: Gordon and Breach). 